\title{
МОРФОЛОГИЯ ПОВЕРХНОСТИ ПЛЕНОК PbTe/Si (100), СИНТЕЗИРОВАННЫХ МОДИФИЦИРОВАННЫМ МЕТОДОМ «ГОРЯЧЕЙ СТЕНКИ»
}

\author{
(C) 2018 А. М. Самойлов ${ }^{1}$, О. Г. Кузьминых ${ }^{1}$ Ю. В. Сыноров ${ }^{2}$, С. А. Ивков ${ }^{1}$, Б. Л. Агапов ${ }^{1}$, \\ Е. К. Белоногов ${ }^{3,1}$ \\ ${ }^{1}$ Воронежский государственный университет, Университетская пл., 1, 394018 Воронеж, Россия \\ e-mail:samoylov@chem.vsu.ru \\ ${ }^{2}$ Воронежский государственный университет инженерных технологий, пр. Революиии, 19, \\ 394036 Воронеж, Россия \\ e-mail:synor@mail.ru \\ ${ }^{3}$ Воронежский государственный технический университет, Московский пр., 14 , \\ 394026, Воронеж, Россия
}

Поступила в редакцию 12.01.2018

\begin{abstract}
Аннотация. Методами растровой электронной микроскопии, локального рентгеноспектрального анализа, рентгенофазового анализа и атомно-силовой микроскопии изучен количественный элементный состав, фазовая природа, а также морфология поверхности гомогенных пленок теллурида свинца, синтезированных модифицированным методом «горячей стенки» на подложках Si (100). При повышении температуры Si (100) подложки до $T=593-608$ K синтезированы мозаичные монокристаллические пленки РdTе с текстурой (100). Установлено, что повышение температуры Si подложки при остальных постоянных режимах синтеза способствует увеличению средних латеральных размеров кристаллитов пленок. Показано, что средняя величина латеральных размеров кристаллитов пленок PbTe на подложках $\mathrm{Si}(100)$ увеличивается с ростом толщины и продолжительности синтеза. Установлено, что средние значения шероховатости пленок $\mathrm{PbTe} / \mathrm{Si}$ (100) увеличиваются с ростом средней толщины пленок PbTe. При этом поликристаллические пленки PbTe/Si (100) при одинаковых значениях средней толщины характеризуются более высокими значениями шероховатости по сравнению с мозаичными монокристаллическими образцами PdTe (100).
\end{abstract}

Ключевые слова: теллурид свинца, тонкие пленки, микроструктура, морфология поверхности, латеральный размер кристаллитов, шероховатость поверхности.

DOI: https://doi.org/10.17308/kcmf.2018.20/483

\section{ВВЕДЕНИЕ}

Повышенный интерес к изучению узкозонных полупроводниковых соединений $\mathrm{A}^{\mathrm{IV}} \mathrm{B}^{\mathrm{VI}}$, в частности, теллурида свинца, обусловлен несколькими основными причинами [1-6]. Прежде всего, соединения $\mathrm{A}^{\mathrm{IV}} \mathrm{B}^{\mathrm{VI}}$ и твердые растворы на их основе являются перспективными материалами для эффективного детектирования инфракрасного (ИК) излучения в интервале длин волн 3-25 мкм [7-8]. Сравнительно недавно для легированных монокристаллов и пленок $\mathrm{PbTe}$ было установлено явление терагерцовой фотопроводимости [9]. Кроме того, благодаря уникальному сочетанию оптимальных значений диэлектрической проницаемости, коэффициен- та Зеебека и высокой теплопроводности в среднем температурном диапазоне теллурид свинца можно применять для производства высокоэффективных термоэлектрических устройств, в особенности для преобразования отработанного тепла в электрическую энергию при промышленном производстве или в автомобильных двигателях [10-12].

При создании планарных ИК-детекторов тонкие пленки РbТе обладают рядом существенных достоинств по сравнению с основными конкурентами - твердыми растворами $\mathrm{Cd}_{1-x} \mathrm{Hg}_{x} \mathrm{Te}$, а также InSb $[8,13]$. В зависимости от природы легирующей примеси на основе $\mathrm{PbTe}$ и его твердых растворов можно создавать как фоторезистивные, так и 
фотовольтаичные ИК-детекторы. Формирование гетероструктур $\mathrm{PbTe} / \mathrm{Si}$ позволяет создавать фокальные ИК-детекторы большой площади, включающие в себя сенсорный материал и прибор цифровой обработки регистрируемого сигнала [13].

В микроэлектронных и оптоэлектронных приборах и устройствах теллурид свинца используется в виде пленок нанометровой толщины, где определяющее значение взаимодействия излучения с материалом играют поверхностные явления. Функциональные характеристики планальных ИК-сенсоров на основе гетероструктур $\mathrm{PbTe} / \mathrm{Si}$ существенным образом зависят от физико-химического состояния их поверхности, в частности, от морфологии и шероховатости [14-15]. Для идеального поглощения детектируемого теплового излучения требуется максимально гладкая поверхность пленки фоточувствительного слоя.

Анализ публикаций, посвященных синтезу пленок полупроводниковых соединений с летучими компонентами, показал, что наиболее эффективными являются методы, основанные на эпитаксии из паровой фазы: молекулярно-лучевая эпитаксия (МЛЭ) и метод «горячей стенки» (ГС) [13, $16,17]$. В сверхвысоком вакууме методом МЛЭ с минимальными скоростями осаждения конденсатов $\sim 30$ нм в минуту (1 монослой/с) выращивают пленки многих материалов [18]. Для формирования пленок с высокой степенью структурного совершенства после непосредственного синтеза методом МЛЭ требуется дополнительный гомогенизирующий отжиг, поскольку синтез осуществляется в условиях, далеких от термодинамического равновесия [15].

При выборе метода формирования тонких пленок и наноструктур на основе $\mathrm{PbTe}$, пригодного для промышленного изготовления ИК-оптоэлектронных приборов или термоэлектрических преобразователей, необходим компромисс между эффективностью, стабильностью и воспроизводимостью функциональных параметров изделий, качеством материалов и себестоимостью технологии их получения. Синтез пленок полупроводниковых соединений с летучими компонентами методом ГС не требует дорогостоящей сверхвысоковакуумной аппаратуры, а также последующего гомогенизирующего отжига, поскольку осуществляется в квазиравновесных условиях $[17,19]$.

Ранее было показано, что пленки $\mathrm{PbTe} \mathrm{и}$ $\mathrm{Pb}_{1-x} \mathrm{Sn}_{x}$ Те, синтезированные методом МЛЭ на подложках $\mathrm{BaF}_{2}$, толщиной менее 500 нм, характеризуются заметным ухудшением функциональных параметров по сравнению с образцами толщиной $\sim 1$ мкм [15]. Кроме того, в работе [20] для пленок $\mathrm{PbTe} / \mathrm{Si}(100)$ констатировали факт снижения подвижности дырок при уменьшении толщины от величин $\sim 2.5$ до 1.0 мкм. Однако авторы $[15,20]$ не обсуждали причину деградации функциональных свойств пленок $\mathrm{PbTe}$ и $\mathrm{Pb}_{1-x} \mathrm{Sn}_{x}$ Те с уменьшением их толщины.

Анализ литературных данных позволяет сделать вывод о том, что до настоящего времени при выращивании пленок $\mathrm{PbTe} / \mathrm{Si}$ (100) методом ГС не уделяли внимания изучению влияния условий синтеза и его продолжительности на морфологию их поверхности.

Целью данной работы является установление характера изменения морфологии поверхности тонких пленок $\mathrm{PbTe}$, полученных модифицированным методом «горячей стенки» на подложках $\mathrm{Si}$ (100), в зависимости от их толщины, продолжительности и технологических условий синтеза (температура подложки и величина парциальных давлений паров свинца и теллура).

\section{ОБЪЕКТЫ И МЕТОДЫ ИССЛЕДОВАНИЯ}

$\mathrm{B}$ настоящей работе синтез пленок РbТе на подложках $\mathrm{Si}$ (100) осуществляли модифицированным методом ГС с использованием графитовой реакционной камеры $[14,17,19]$. Используемая в работе технология синтеза пленок РbТе сочетает в себе преимущества двух методов: выращивание пленки в квазиравновесных условиях (классический метод ГС) и формирование контролируемых молекулярных потоков летучих компонентов из независимых источников (метод молекулярно-лучевой эпитаксии - МЛЭ) [14]. В качестве исходных компонентов использовали металлический свинец марки «0000» ЭКСТРА и теллур марки ОСЧ-9-5, Непосредственно перед синтезом пленок РbTe исходные компоненты очищали от оксидов перегонкой в вакууме.

Для синтеза пленок теллурида свинца реакционную камеру вакуумировали до остаточного давления $5 \cdot 10^{-4}$ Па. Для удаления адсорбированных молекул газообразных веществ перед процессом напыления пленок теллурида свинца $\mathrm{Si}$ подложку нагревали до температуры $T_{\text {sub }} \sim 720-730 \mathrm{~K}$ и выдерживали в таких условиях в течение 10 минут.

Вследствие восстановительного характера остаточной атмосферы и геттерирования газов графитовыми стенками камеры при испарении вещества парциальное давление кислорода в реакцион- 
ном объеме снижается до уровня $5 \cdot 10^{-7}$ Па [8]. При этом в камере реализуются условия, соответствующие парциальному давлению остаточных газов на уровне $10^{-7}$ Па, что обеспечивает возможность удаления естественного оксида $\mathrm{SiO}_{2}$ с поверхности $\mathrm{Si}$ подложки непосредственно перед осаждением конденсата бинарного полупроводника. Для удаления пленки естественного оксида $\mathrm{SiO}_{2}$ с поверхности кремниевых пластин марок КДБ-200 и КЭФ-200 использовали метод, при котором поверхность подложки выдерживалась в парах теллура в течение 20-30 минут непосредственно перед осаждением полупроводника $[8,17]$.

Одной из центральных задач данной работы являлось исследование влияния технологических условий синтеза на микроструктуру и морфологию поверхности пленок $\mathrm{PbTe} / \mathrm{Si}(100)$. С этой целью в процессе синтеза пленок теллурида свинца температуру $\mathrm{Si}$ (100) подложки изменяли в интервале $T_{\text {sub }}=553 \pm 2-623 \pm 2 \mathrm{~K}$. Величину давления паров летучих компонентов в реакционной камере модифицированного метода ГС варьировали в следующих пределах: парциальное давление свинца $p_{\mathrm{Pb}}=6.83-17.123$ Па, парциальное давление теллура $p_{\text {Tе }}=0.0085-0.0368$ Па. Длительность процесса синтеза образцов $\mathrm{PbTe} / \mathrm{Si}$ (100) составляла $15-120$ минут.

Количественный элементный состав синтезированных пленок $\mathrm{PbTe}$ контролировали методом локального рентгеноспектрального анализа (ЛРСА) с использованием приборов JEOL JCM 6380 LV (спектрометр с энергетической дисперсией) и JEOL JCA 840 (спектрометр с волновой дисперсией). По данным ЛРСА все синтезированные методом ГС пленки $\mathrm{PbTe}$ характеризуются количественным составом, соответствующим стехиометрическому соотношению компонентов в пределах области гомогенности бинарного соединения [20].

Кристаллическую структуру исходных подложек $\mathrm{Si}(100)$, а также синтезированных пленок $\mathrm{PbTe}$ характеризовали методом рентгеновской дифрактометрии на приборе ДРОН-4-07 с использованием $\mathrm{Co} K_{\alpha}$ и $\mathrm{Cu} K_{\alpha}$-излучения. Индицирование рентгеновских рефлексов подложек $\mathrm{Si}$, а также пленок $\mathrm{PbTe}$ проводили на основе международной базы данных [21].

Поверхность и свежеприготовленные сколы синтезированных гетероструктур $\mathrm{PbTe} / \mathrm{Si}$ (100) изучали методом растровой электронной микроскопии (РЭМ) на приборе JEOL JCM 6380 LV.

Среднюю скорость роста $\bar{v}$ и погрешность $\Delta v$ пленок $\mathrm{PbTe}$ рассчитывали по формуле:

$$
\bar{v} \pm \Delta v=\frac{\bar{d} \pm \Delta d}{\tau}
$$

где $\bar{d}$ - средняя толщина пленки $\mathrm{PbTe}, \Delta d-$ среднеквадратичная погрешность определения толщины; $\tau$ - продолжительность синтеза.

Величину $\bar{d}$ определяли по результатам 15 измерений толщины пленки $\mathrm{PbTe}$ для различных участков изображений сколов гетероструктур $\mathrm{PbTe} / \mathrm{Si}$ (100), полученных методом РЭМ на приборе JEOL JCM 6380 LV (рис. 1).

Также поверхность пленок теллурида свинца исследовали методом атомно-силовой микроскопии (ACM) на приборе Solver P47 Pro. Для этого анализировали фрагменты поверхности в форме квадрата со сторонами $6 \times 6$ мкм. Шероховатость поверхности $R_{\mathrm{a}}$ - как среднеквадратичное отклонение от средней высоты.

\section{ЭКСПЕРИМЕНТАЛЬНЫЕ РЕЗУЛЬТАТЫ И ИХ ОБСУЖДЕНИЕ}

Согласно экспериментальным данным, полученным методом РФА, все синтезированные образцы $\mathrm{PbTe} / \mathrm{Si}$ (100) можно разделить на две группы (рис. 2). Как показано на рис. $2 a$, первую из них составляют пленки теллурида свинца с мозаичной монокристаллической структурой и ориентацией (100). Ко второй группе следует отнести поликристаллические образцы (рис. 2b), на дифракционных картинах которых при преимущественной интенсивности рефлексов теллурида свинца $(h 00)$ присутствую слабые смешанные рефлексы.

Анализ всех РЭМ-изображений (рис. 1) сколов полученных образцов $\mathrm{PbTe} / \mathrm{Si}$ (100) показал, что в зависимости от технологических режимов и продолжительности синтеза, которая составляла $15,30,45,60,90$ и 120 минут, толщина пле-

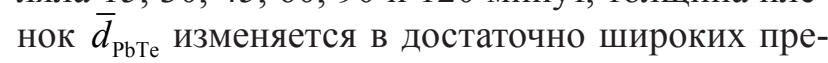
делах: от $0.084 \pm 0.005$ мкм до $1.85 \pm 0.04$ мкм. Установлено, что погрешность определения толщины для пленок с ярко выраженной текстурой (100) существенно ниже, чем для поликристаллических образцов.

Сопоставление данных РФА с результатами определения толщины, а также с результатами расчетов средней скорости роста $\bar{v}$ пленок $\mathrm{PbTe} / \mathrm{Si}$ (100) в зависимости от технологических условий процесса синтеза модифицированным методом ГС позволили объяснить причину формирования поликристаллических и монокристаллических образцов. Как показано на рис. 3, поликристаллические пленки $\mathrm{PbTe} / \mathrm{Si}$ (100) формируются при минималь- 
a)

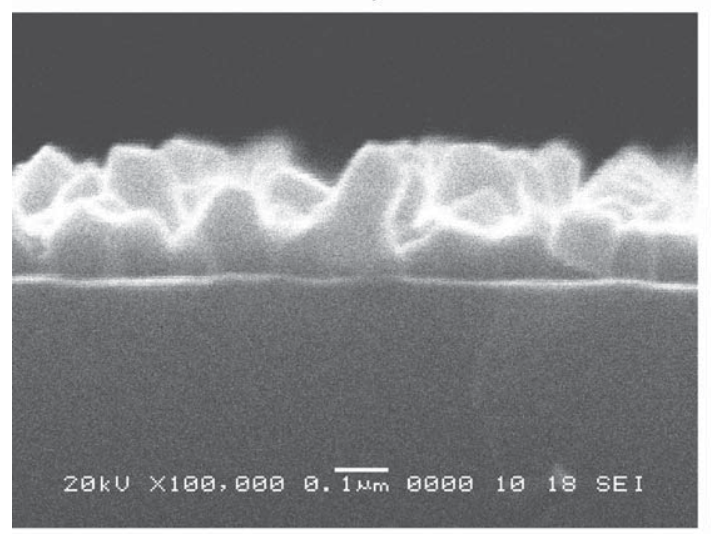

c)

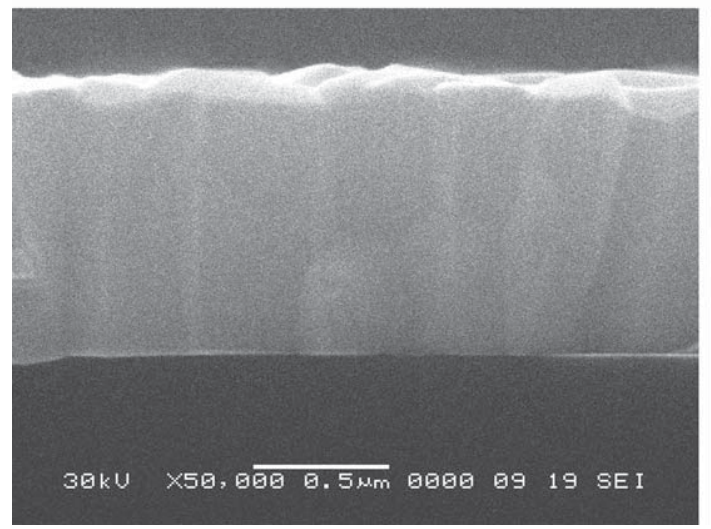

b)

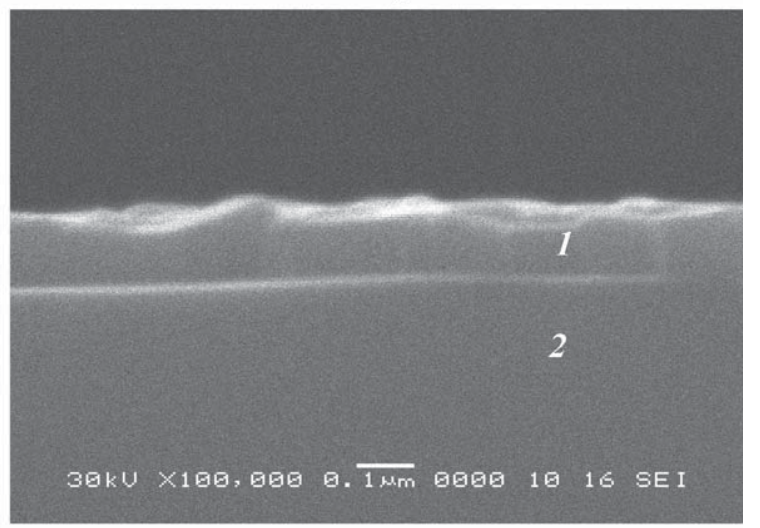

d)

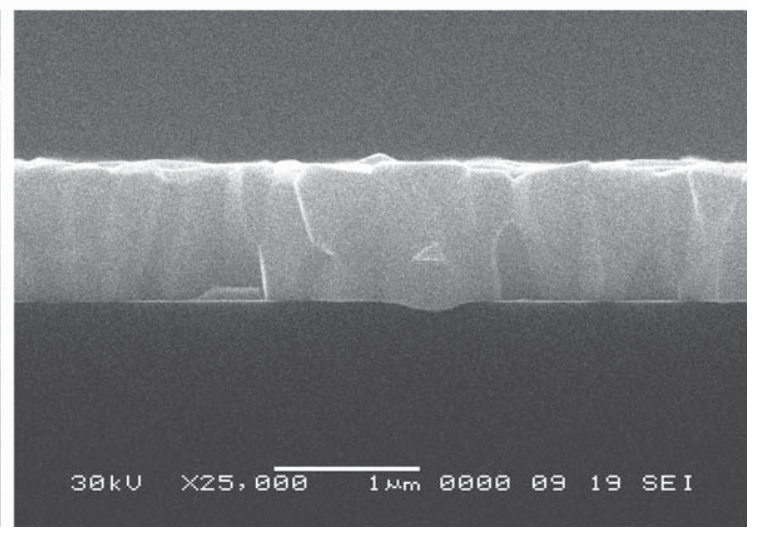

Рис. 1. РЭМ микрофотографии сколов пленок РbТе на подложках $\mathrm{Si}(100)$ при различном увеличении: $a, b-$ увеличение $\times 100000 ; c$ - увеличение $\times 50000 ; d$ - увеличение $\times 25000 ; a, d$ - поликристаллические пленки; $b, c-$ мозаичные монокристаллические пленки с текстурой (100); 1 - пленка $\mathrm{PbTe} ; 2$ - подложка Si (100)

[Fig. 1. SEM micrographs of $\mathrm{PbTe} / \mathrm{Si}$ (100) films cross-sections at various magnification: $a, b-\times 100000$ magnification; $c-\times 50000$ magnification; $d-\times 25000$ magnification; $a, d$-polycrystalline films; $b, c-$ mosaic single crystal films with (100) texture; 1 - PbTe film; 2 - Si (100) substrate]

ных температурах подложки $T_{\text {sub }}=553 \mathrm{~K}$, а также высоких значения парциальных давлений летучих компонентов - свинца и теллура. При этих режимах синтеза отклонение от условий термодинамического равновесия является весьма существенным, вследствие чего реализуются максимальные скорости роста конденсата из паровой фазы (рис. 3). Как видно на рис. 3 (кривая 2 и кривая 3), при постоянных условиях синтеза увеличение температуры подложки от $T_{\text {sub }}=553 \mathrm{~K}$ до $T_{\text {sub }}=623 \mathrm{~K}$ приводит к снижению средней скорости $\bar{v}$ формирования конденсата практически в два раза. Кроме того, полученные данные подтверждают сделанное ранее предположение о том, что скорость роста пленок PbTe модифицированным методом ГС изменяется в процессе синтеза [20]. Сопоставление результатов расчетов для пленок $\mathrm{PbTe} / \mathrm{Si}$ (100), выращенных при одинаковых условиях в течение 60 минут и 120 минут, показало, что значения $\bar{v}$ несколько выше, если длительность процесса составляет 60 минут (рис. 3, кривая 1 и кривая 2).

На следующем этапе методом РЭМ была изучена морфология поверхности мозаичных монокристаллических и поликристаллических пленок $\mathrm{PbTe} / \mathrm{Si}$ (100). Чтобы свести к минимуму возможные случайные погрешности были получены РЭМ-изображения поверхности пленок в трех различных местах при трех различных увеличениях: $\times 100000, \times 50000$ и $\times 25000$ (рис. 4). Таким образом, от каждого образца было получено девять массивов данных.

При изучении морфологии поверхности пленок теллурида свинца на подложках $\mathrm{Si}$ (100) определяли средний латеральный размер кристаллитов $\bar{l}_{\text {крист. }}$. Методом математической статистики по изображениям, полученным при различном увеличении (рис. 4), рассчитывали наиболее вероятные значения $\bar{l}_{\text {крист. }}$ для каждой пленки $\mathrm{PbTe} / \mathrm{Si}(100)$. 

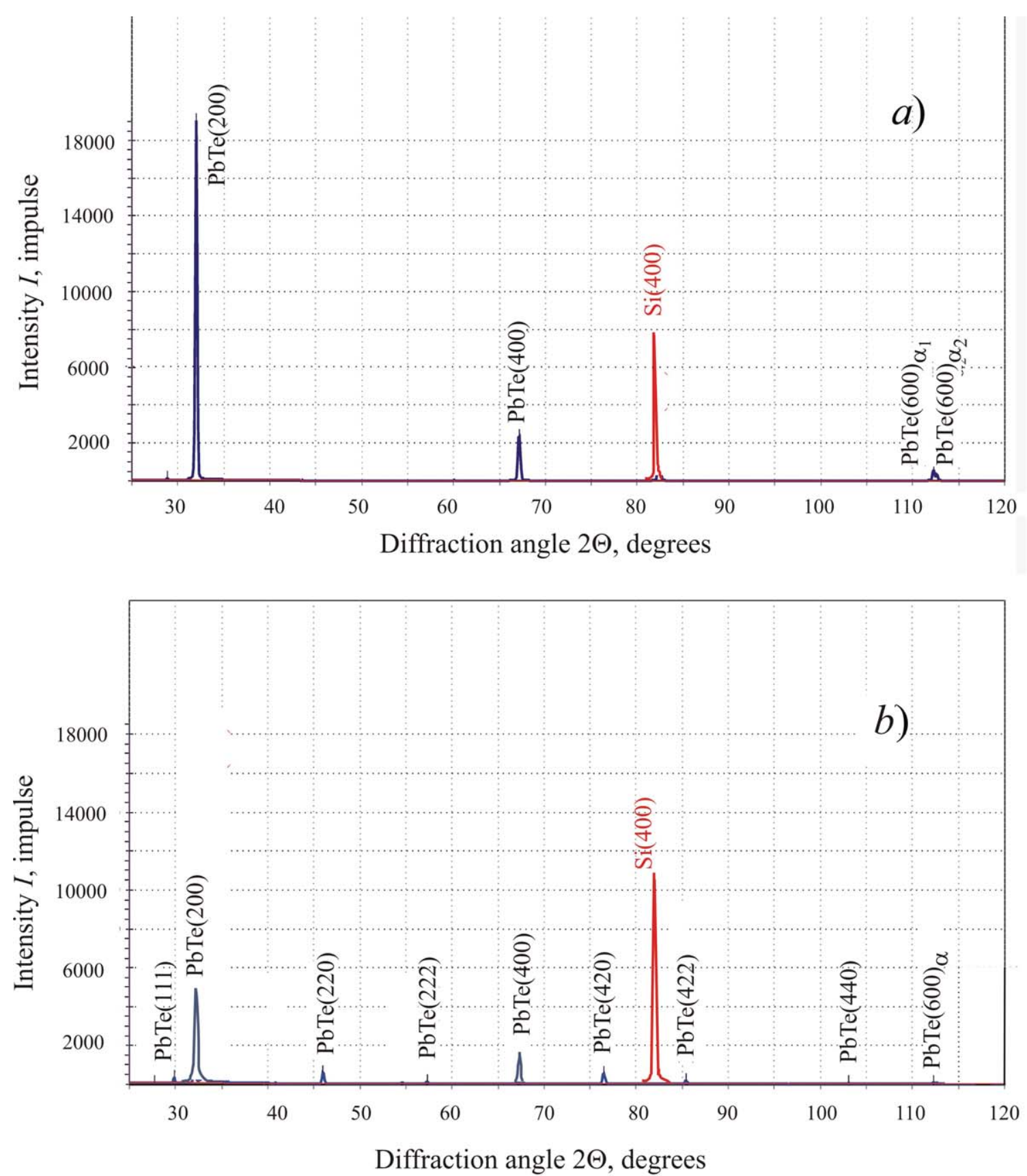

Рис. 2. Дифрактограммы пленок РbTe, синтезированных на подложках $\mathrm{Si}$ (100) модифицированным методом $\Gamma \mathrm{C}: a)$ мозаичный монокристаллический образец с текстурой $(100) ; b$ поликристаллический образец [Fig. 2. X-ray diffraction patterns of PbTe films prepared on Si (100) substrates by «hot wall» epitaxy technique: a) mosaic single crystal film with (100) texture; $b$ - polycrystalline film] 


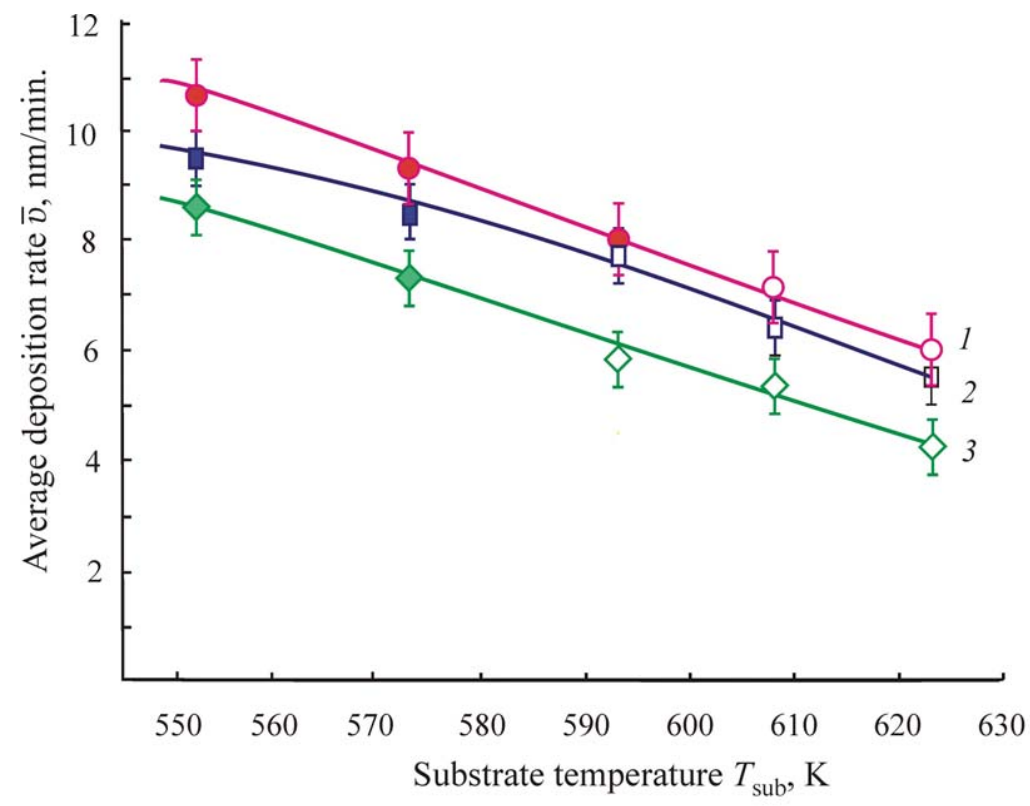

Рис. 3. Зависимость скорости роста пленок $\mathrm{PbTe} / \mathrm{Si}$ (100) от температуры подложки: 1 - продолжительность синтеза $\tau=60$ минут; 2, 3 - продолжительность синтеза $\tau=120$ минут; $1,2-$ синтез при давлении паров свинца $p_{\mathrm{Pb}}=17.123$ Па и паров теллура $p_{\mathrm{Te}}=0.0368$ Па; 3 - синтез при давлении паров свинца $p_{\mathrm{Pb}}=6.83$ Па и паров теллура $p_{\text {те }}=0.0085$ Па

[Fig. 3. Dependence of average deposition rate of $\mathrm{PbTe} / \mathrm{Si}$ (100) films upon substrate temperature: 1 - synthesis duration $\tau=60$ minutes; 2, 3- synthesis duration $\tau=120$ minutes; $1,2-$ synthesis under lead vapour pressure $p_{\mathrm{Pb}}=17.123 \mathrm{~Pa}$ and tellurium vopour pressure $p_{\mathrm{Te}}=0.0368 \mathrm{~Pa} ; 3$ - synthesis lead vopour pressure $p_{\mathrm{Pb}}=6.83 \mathrm{~Pa}$ and tellurium vapour pressure $\left.p_{\mathrm{Te}}=0.0085 \mathrm{~Pa}\right]$

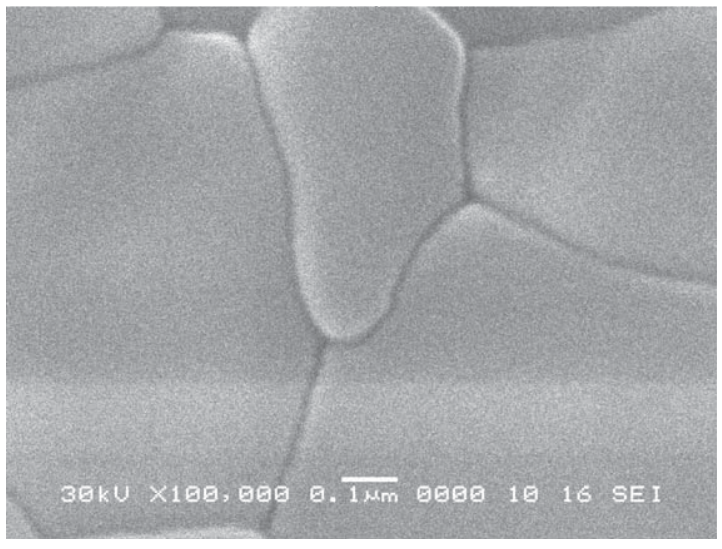

a)

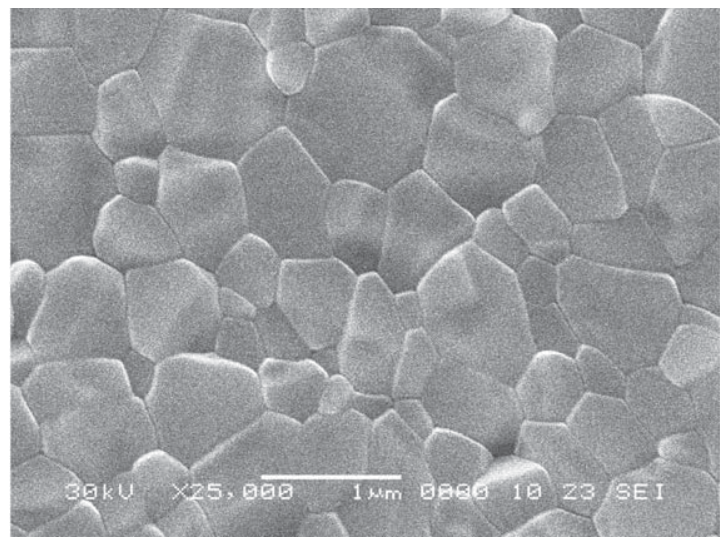

c)

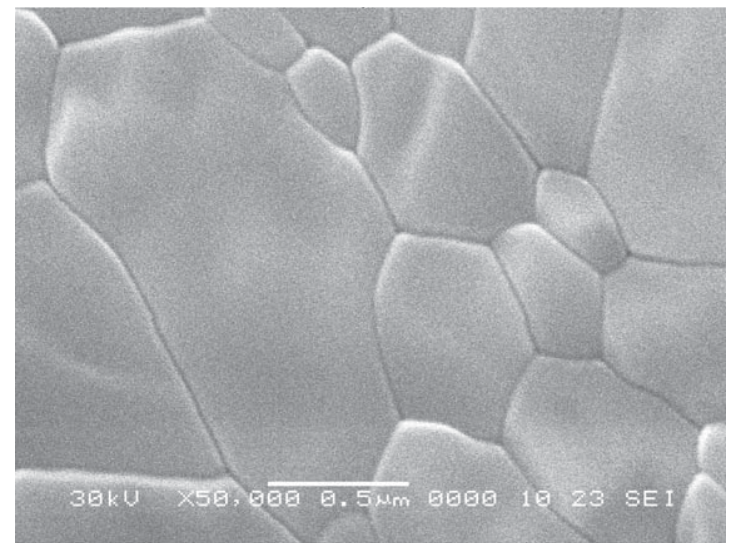

b)

Рис. 4. РЭМ-изображения поверхности пленок $\mathrm{PbTe} / \mathrm{Si}(100)$ при различном увеличении: $a$ ) $\times 100000 ; b)-\times 50000 ; c)-\times 25000$.

[Fig. 4. SEM micrograph images of $\mathrm{PbTe} / \mathrm{Si}$ (100) film top side under various magnification: $a$ ) $\times 100000$ magnification; $b) \times 50000$ magnification; c) $\times 25000$ magnification] 
Результаты расчетов наиболее вероятных средних латеральных размеров кристаллитов пленок $\mathrm{PbTe} / \mathrm{Si}$ (100) представлены в виде гистограмм на рис. 5. При этом установлено, что суммарная вероятность образования кристаллитов, латеральные размеры которых соответствуют среднему значению, составляет от 30 до $60 \%$. Необходимо отметить, что пленки $\mathrm{PbTe} / \mathrm{Si}$ (100) с мозаичной монокристаллической структурой характеризуются меньшим разбросом значений $\bar{l}_{\text {крист. }}$ и более равномерным их распределением (рис. $5 a$ ) по сравнению с поликристаллическими образцами (рис. $5 b$ ). Увеличение погрешности в оценке величины $\bar{l}_{\text {крист. }}$ для поликристаллических образцов можно объяснить тем, что кристаллиты, формирующие такие пленки, могут располагаться под разными углами относительно поверхности подложки.

Весьма важной является информация о характере изменения средних латеральных размеров кристаллитов $\bar{l}_{\text {крис. }}$ синтезированных пленок $\mathrm{PbTe} / \mathrm{Si}$ (100) в зависимости от величины средней толщины $\bar{d}_{\text {Ръте }}$ и условий синтеза (температура подложки $T_{\text {sub }}$ ), которая представлена на рис. 6. Как видно на этом рисунке, для всех синтезированных образцов $\mathrm{PbTe} / \mathrm{Si}(100)$ независимо от режимов получения наблюдается общая тенденция увеличения средних латеральных размеров кристаллитов с ростом средней толщины пленки. Необходимо отметить, что пленки $\mathrm{PbTe} / \mathrm{Si}$ (100), синтезированные при темпе- ратуре подложки $T_{\text {sub }}=608 \pm 2 \mathrm{~K}$, характеризуются более крупными кристаллитами по сравнению с образцами, синтезированными при аналогичных технологических условиях при температуре подложки $T_{\text {sub }}=553 \pm 2 \mathrm{~K}$ (рис. 6, кривая 2).

В дополнение к исследованиям, выполненным методом РЭМ, морфологию поверхности пленок $\mathrm{PbTe} / \mathrm{Si}$ (100) изучали при помощи АCM. Результаты исследования представлены на рис. 7. Анализ результатов статистической математической обработки экспериментальных данных, полученных методом АСМ, позволяет отметить удовлетворительную корреляцию результатов оценки средних размеров кристаллитов $\bar{l}_{\text {крист. }}$. Данные, полученные двумя методами, различаются между собой не более, чем на $10 \%$.

При этом, как видно на рис. $7 a$, погрешность

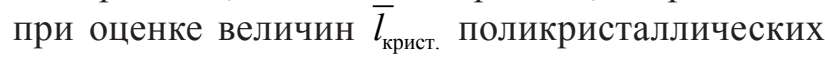
образцов РbТе обусловлена тем, что относительно поверхности подложки Si (100) кристаллиты ориентированы под разными углами, в то время как кристаллиты образцов с текстурой (100) имеют разориентацию только относительно оси текстуры (рис. $7 b$ ).

Изучение поверхности пленок $\mathrm{PbTe} / \mathrm{Si}$ (100) методом ACM позволяет получить информацию о ее средней шероховатости $S_{\mathrm{a}}$. Эта информация важна при использовании пленок теллурида свинца в качестве активных элементов оптоэлектрон-

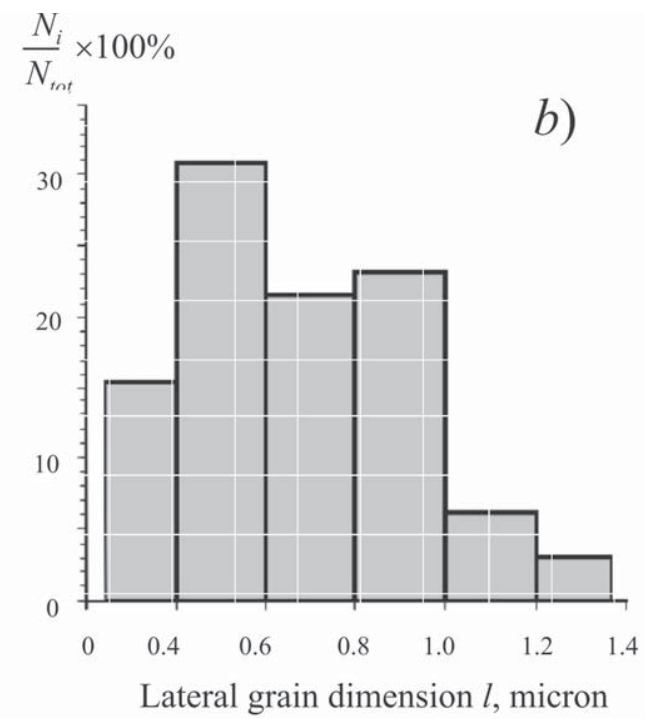

Рис. 5. Гистограммы, иллюстрирующие вероятность распределения латеральных размеров пленок РbТе на подложках $\mathrm{Si}(100): a$ ) образец с мозаичной монокристаллической структурой (толщина $d_{\mathrm{PbTe}}=0.26$ мкм); b) поликристаллический образец (толщина $d_{\mathrm{PbTe}}=1.18$ мкм)

[Fig. 5. Histograms illustrating the lateral dimension distribution of PbTe films on $\mathrm{Si}(100)$ substrates: $a$ ) sample with mosaic single-crystal structure (thickness $d_{\mathrm{PbTe}}=0.26$ microns); $b$ ) polycrystalline sample (thickness of $d_{\mathrm{PbTe}}=1.18$ microns)] 


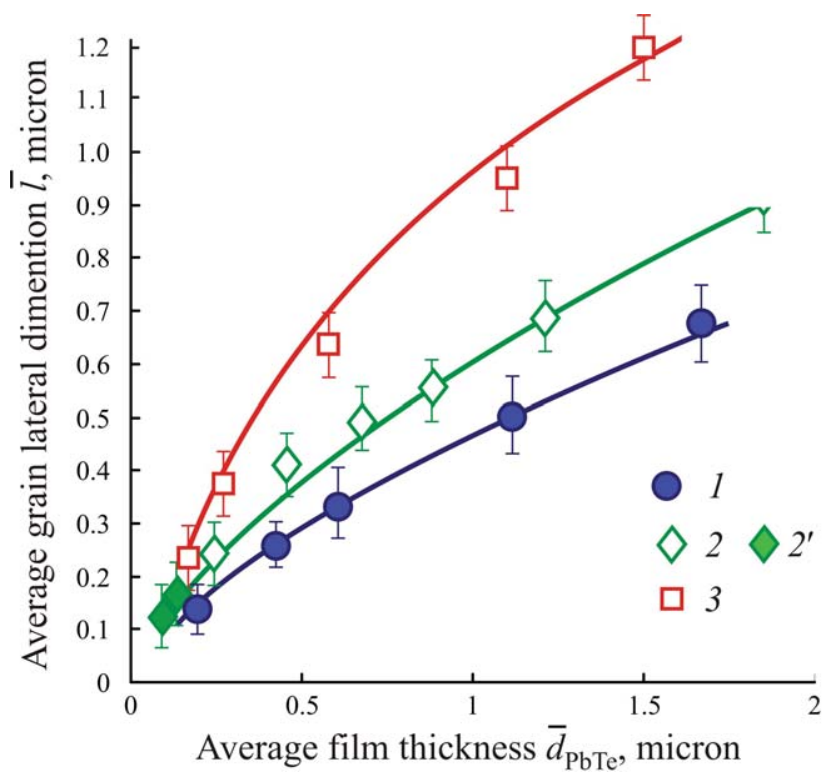

Рис. 6. Зависимость среднего латерального размера кристаллитов $\bar{l}$ пленок $\mathrm{PbTe} / \mathrm{Si}(100)$ от средней толщины пленки $\bar{d}_{\mathrm{PbTe}}: 1$ - температура подложки

$T_{\text {sub }}=553 \pm 2 \mathrm{~K} ; 2$ - температура подложки

$T_{\text {sub }}^{\text {sub }}=573 \pm 2 \mathrm{~K} ; 3$ - температура подложки

$T_{\text {sub }}=608 \pm 2 \mathrm{~K} ; 1,2^{\prime}-$ поликристаллические образцы;

2, 3 - мозаичные монокристаллические образцы

$$
\text { с текстурой (100) }
$$

[Fig. 6. Dependence of the average lateral size $\bar{l}$ of $\mathrm{PbTe} / \mathrm{Si}$ (100) film crystalline grain upon the average thickness $\bar{d}_{\mathrm{PbTe}}: 1-$ substrate temperature $T_{\text {sub }}=553 \pm 2 \mathrm{~K}$; 2 - substrate temperature $T_{\text {sub }}=573 \pm 2 \mathrm{~K} ; 3$ - substrate temperature $T_{\text {sub }}=608 \pm 2 \mathrm{~K} ; 1,2^{\prime}-$ polycrystalline samples; 2, 3 - mosaic single crystal samples with (100) texture]

\section{a)}

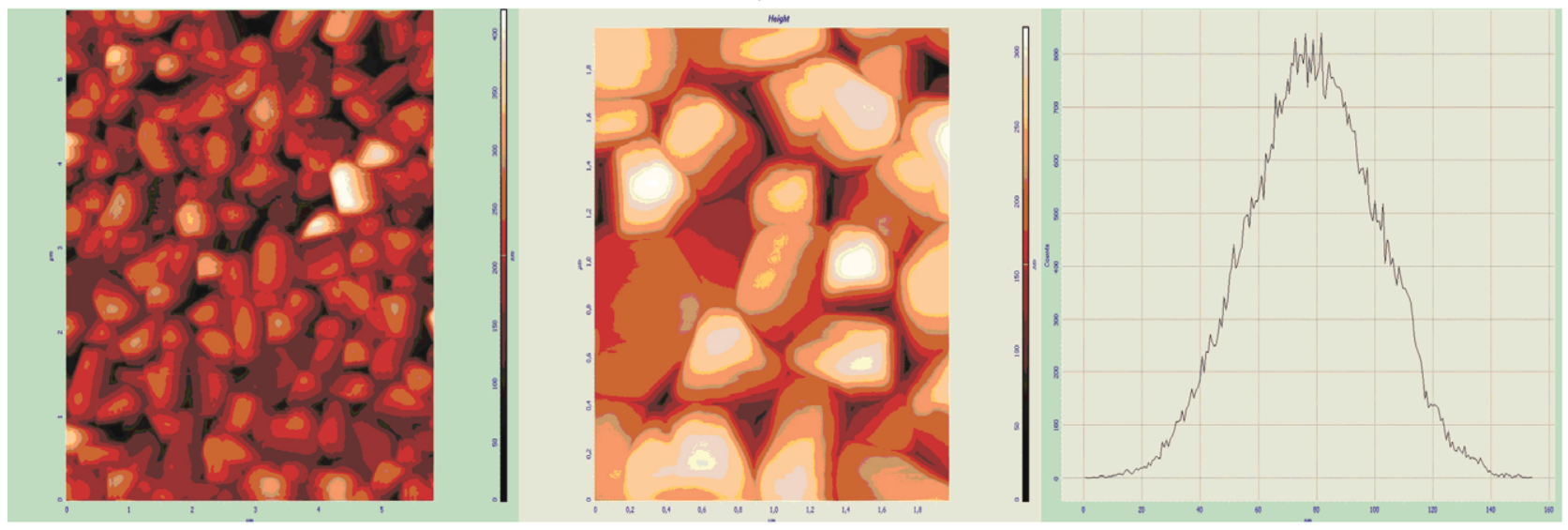

b)
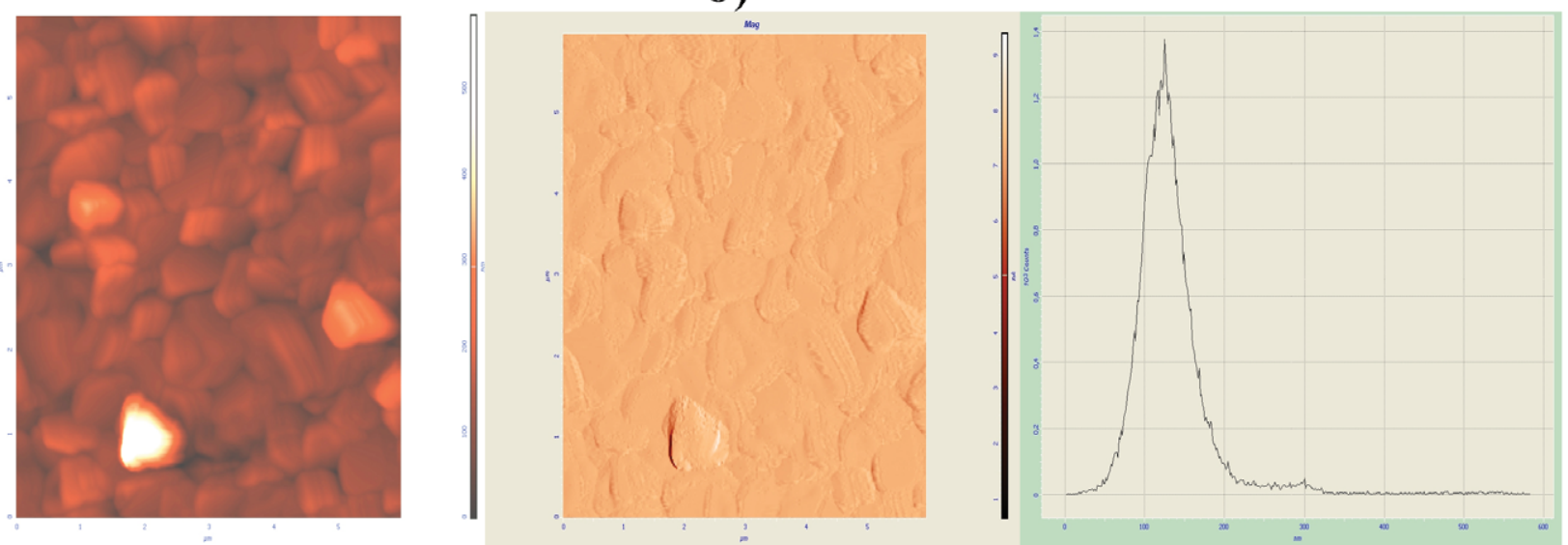

Рис. 7. АСМ-изображения поверхности и средняя шероховатость поверхности пленок $\mathrm{PbTe} / \mathrm{Si}(100)$ : $a$ ) поликристаллический образец; $b$ ) мозаичный монокристаллический образец с текстурой (100)

[Fig. 7. Atomic force microscopy (AFM) images and average surface roughness of $\mathrm{PbTe} / \mathrm{Si}$ (100) film: $a$ ) polycrystalline sample; $b$ ) mosaic single-crystal sample with (100) texture] 
ных приборов (ИК-детекторов и лазерных светодиодов). После статистической обработки результаты полученных экспериментальных данных в зависимости от толщины пленок $\bar{d}_{\mathrm{PbTe}}$ представлены на рис. 8.

Как видно на этом рисунке, поликристаллические образцы $\mathrm{PbTe}$, синтезированные при низкой температуре подложки, характеризуются более высокими значениями шероховатости $R_{\mathrm{a}}$ (рис. $8, \kappa p u$ вая 1) по сравнению с мозаичными монокристаллическими пленками $\mathrm{PbTe}$, имеющими строго выраженную текстуру (100) (рис. 8, кривая 2 и кривая 3). При этом для всех синтезированных пленок $\mathrm{PbTe} / \mathrm{Si}$ (100) наблюдается увеличение средних абсолютных значений шероховатости $R_{\mathrm{a}}$ с ростом толщины пленки $\bar{d}_{\mathrm{PbTe}}$. Однако рост значений $R_{\mathrm{a}}$ для поликристаллических и мозаичных монокристаллических пленок $\mathrm{PbTe}$ с текстурой (100) осуществляется по-разному. Для поликристаллических образцов $\mathrm{PbTe} / \mathrm{Si}(100)$ наблюдается монотонное уве-

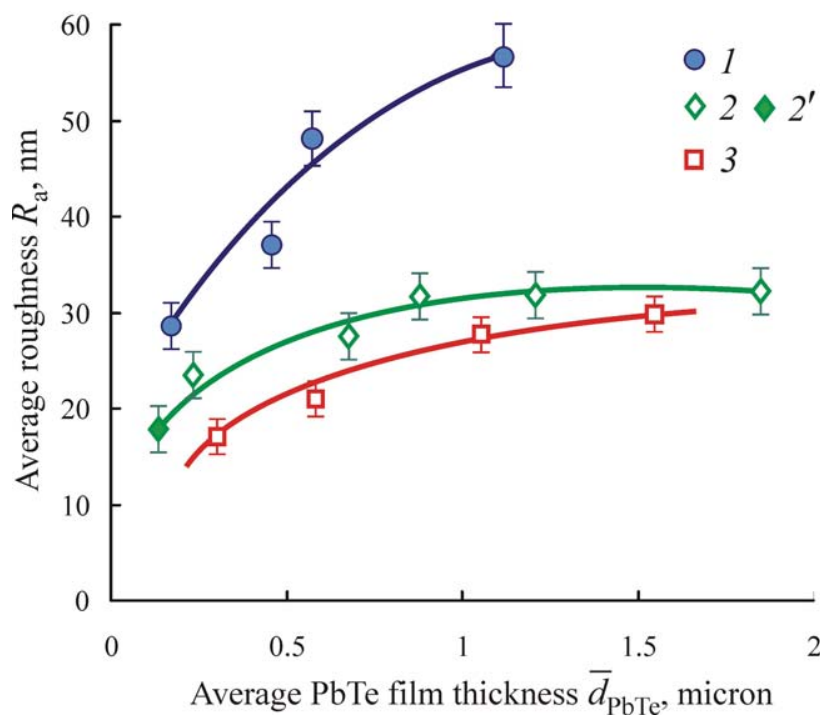

Рис. 8. Зависимость средней величины шероховатости поверхности $R_{\mathrm{a}}$ пленок $\mathrm{PbTe} / \mathrm{Si}$ (100), синтезированных при различной температуре подложки $T_{\text {sub }}$, от их средней толщины $\bar{d}_{\mathrm{PbTe}}: 1$ - температура подложки

$T_{\text {sub }}=553 \pm 2 \mathrm{~K} ; 2-$ температура подложки

$T_{\text {sub }}=573 \pm 2 \mathrm{~K} ; 3$ - температура подложки $T_{\text {sub }}=608 \pm 2 \mathrm{~K} ; 1,2^{\prime}$ - поликристаллические образцы; 2, 3 - мозаичные монокристаллические образцы с текстурой (100)

[Fig. 8. Dependence of average surface roughness $R_{\mathrm{a}}$ of $\mathrm{PbTe} / \mathrm{Si}$ (100) films synthesized at different substrate temperature $T_{\text {sub }}$ upon their average thickness $\bar{d}_{\mathrm{PbTe}}: 1-$ substrate temperature $T_{\text {sub }}=553 \pm 2 \mathrm{~K} ; 2$ - substrate temperature $T_{\text {sub }}=573 \pm 2 \mathrm{~K} ; 3$ - substrate temperature $T_{\text {sub }}=608 \pm 2 \mathrm{~K} ; 1,2^{\prime}-$ polycrystalline samples; $2,3-$ mosaic single-crystal samples with (100) texture] личение средней шероховатости $R_{\mathrm{a}}$ с ростом толщины пленки для всего изученного интервала толщин (рис. 8, кривая 1). Для образцов $\mathrm{PbTe} / \mathrm{Si}$ (100) с текстурой (100) наблюдается более пологий рост значений $R_{\mathrm{a}}$ с увеличением $\bar{d}_{\mathrm{PbTe}}$. Кроме того, для пленок $\mathrm{PbTe} \mathrm{с} \mathrm{текстурой} \mathrm{(100)} \mathrm{при} \mathrm{достижении} \mathrm{тол-}$ щин $\bar{d}_{\mathrm{PbTe}}>0.8$ мкм значения шероховатости практически не изменяются с ростом толщины (рис. 8 , кривая 2 и кривая 3).

В настоящей работе в качестве одного из критериев, который в определенной степени позволяет охарактеризовать вклад в функциональные свойства пленок составляющей от поверхности, предложено отношение средней шероховатости поверхности к средней толщине пленки $\left(R_{\mathrm{a}} / \bar{d}_{\mathrm{PbTe}}\right)$, если обе величины представлены в одинаковой размерности. Безусловно, вклад поверхности в общую картину физико-химических свойств зависит от целого комплекса параметров, в частности, от длины экранирования Дебая $\lambda_{\mathrm{D}}$. В зависимости от того, в каких устройствах планируется использование пленок тех или иных материалов, соотношение вкладов поверхности и объема в функциональные свойства может изменяться в широких пределах. Например, в целях повышения чувствительности газовых сенсоров на основе пленок широкозонных металлоксидных полупроводников требуется максимальная развитая поверхность функциональных слоев [22].

Результаты проведенных расчетов отношения $R_{\mathrm{a}} / \bar{d}_{\mathrm{PbTе}}$ для пленок $\mathrm{PbTe}$, синтезированных модифицированным методом ГС, в зависимости от их толщины представлены на рис. 9. При анализе данного рисунка очевиден факт уменьшения значений $R_{\mathrm{a}} / \bar{d}_{\mathrm{PbTe}}$ с ростом толщины синтезированных пленок $\mathrm{PbTe}$ независимо от степени их структурного совершенства. При этом максимальными значениями $S_{\mathrm{a}} / \bar{d}_{\mathrm{PbTe}}$ характеризуются поликристаллические образцы, синтезированные при температуре подложки $T_{\text {sub }}=553 \pm 2 \mathrm{~K}$ (рис. $2 b$ ). Пленки РbТе с мозаичной кристаллической структурой (рис. $2 a$ ), синтезированные при $T_{\text {sub }}=573 \pm 2 \mathrm{~K}$ и $T_{\text {sub }}=608 \pm 2 \mathrm{~K}$, обладают существенно меньшими значениями $R_{\mathrm{a}} / \bar{d}_{\mathrm{PbTе}}$ (рис. 9, кривые 2 и 3 ). Кроме того, для таких образцов при толщинах $\bar{d}_{\mathrm{PbTe}}>1.5$ мкм значения $R_{\mathrm{a}} / \bar{d}_{\mathrm{PbTе}}$ достигают величины $\sim 0.02$ и далее практически не изменяются с ростом толщины пленки. Принимая во внимание факт постоянства значений $R_{\mathrm{a}} / \bar{d}_{\mathrm{PbTe}}$, можно предположить, что для пленок РbТе с мозаичной монокристаллической текстурой (100) толщиной более 1.2 мкм объемные свойства будут преобладать над поверхностными и будут обеспе- 


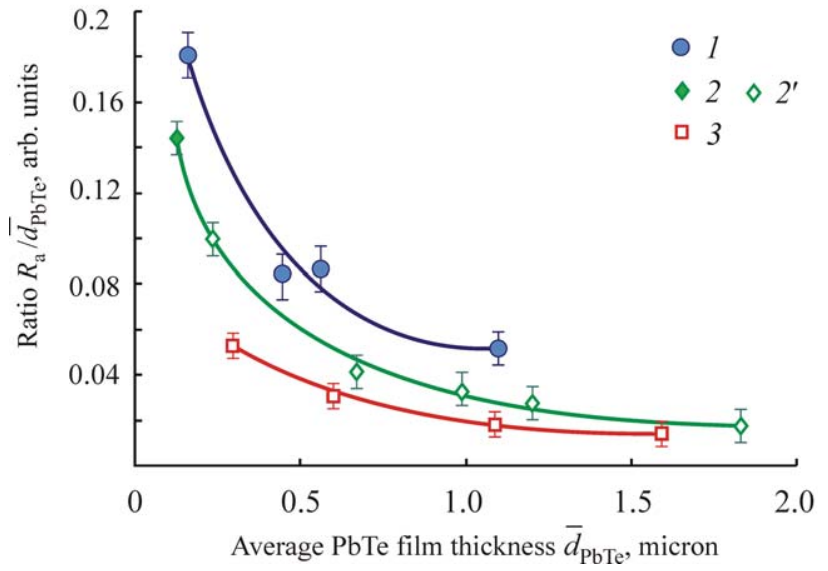

Рис. 9. Зависимость отношения шероховатости поверхности $R_{\mathrm{a}}$ к средней толщине пленок $\mathrm{PbTe} / \mathrm{Si}$ (100), синтезированных при различной температуре подложки $T_{\mathrm{sub}}$, от их средней толщины $\bar{d}_{\mathrm{PbTe}}: 1-$ температура подложки $T_{\text {sub }}=553 \pm 2 \mathrm{~K} ; 2$ - температура подложки $T_{\text {sub }}=573 \pm 2 \mathrm{~K} ; 3$ - температура подложки $T_{\text {sub }}=608 \pm 2 \mathrm{~K} ; 1,2^{\prime}-$ поликристаллические образцы; 2, 3 - мозаичные монокристаллические образцы с текстурой (100)

[Fig. 9. Dependence of $R_{\mathrm{a}} / \bar{d}_{\mathrm{PbTe}}$ ratio of $\mathrm{PbTe} / \mathrm{Si}(100)$ films synthesized at different substrate temperature $T_{\text {sub }}$ upon their average thickness $\bar{d}_{\mathrm{PbTe}}: 1$ - substrate temperature $T_{\text {sub }}=553 \pm 2 \mathrm{~K} ; 2$ - substrate temperature $T_{\text {sub }}=573 \pm 2 \mathrm{~K} ; 3-$ substrate temperature $T_{\text {sub }}=608 \pm 2 \mathrm{~K}$;

$1,2^{\prime}$ - polycrystalline samples; 2, 3 - mosaic singlecrystal samples with (100) texture]

чивать главный вклад в величину функциональных параметров. Данное предположение целесообразно проверить детальным изучением изменения концентрации носителей заряда и их подвижности в зависимости от толщины пленок $\mathrm{PbTe}$, синтезированных модифицированным методом ГС, что планируется в последующих исследованиях.

\section{ВЫВОДЫ}

1. Установлено, что пленки $\mathrm{PbTe,} \mathrm{синтезиро-}$ ванные модифицированным методом «горячей стенки» на подложках Si (100), при температуре подложки $T_{\text {sub }} \leq 553 \mathrm{~K}$ являются поликристаллическими, в то время как повышение температуры подложки $T_{\text {sub }} \geq 573 \mathrm{~K}$ приводит к формированию монокристаллических мозаичных пленок с текстурой (100).

2. Методами РЭМ и АСМ установлено, что средняя величина латеральных размеров кристаллитов пленок $\mathrm{PbTe} / \mathrm{Si}$ (100) увеличивается с ростом толщины и продолжительности синтеза.
3. Методами РЭМ и АСМ установлено, что повышение температуры подложки при остальных постоянных режимах синтеза способствует увеличению средних латеральных размеров кристаллитов пленок $\mathrm{PbTe}$, синтезированных модифицированным методом «горячей стенки» на подложках $\mathrm{Si}(100)$.

4. Методом АСМ было установлено, что шероховатость поверхности пленок $\mathrm{PbTe} / \mathrm{Si}$ (100) увеличивается с ростом их средней толщины, при этом поликристаллические пленки $\mathrm{PbTe} / \mathrm{Si}$ (100) при одинаковых значениях средней толщины характеризуются более высокими значениями шероховатости по сравнению с мозаичными монокристаллическими образцами.

5. В качестве критерия, позволяющего охарактеризовать вклад в функциональные свойства пленок составляющей от поверхности, предложено отношение средней шероховатости поверхности к средней толщине пленки $\left(R_{\mathrm{a}} / \bar{d}_{\mathrm{PbTe}}\right)$.

6. Установлено, что пленки РbТе с мозаичной кристаллической структурой обладают существенно меньшими значениями $R_{\mathrm{a}} / \bar{d}_{\mathrm{PbTe}}$, при этом для таких образцов при толщинах $\bar{d}_{\mathrm{PbTe}}>1.2$ мкм значения $R_{\mathrm{a}} / \bar{d}_{\mathrm{PbTe}}$ достигают величины $\sim 0.02$ и далее практически не изменяются с ростом толщины пленки.

Экспериментальные исследования были проведены с помощьюю научно-технической базы ЦКПНО ВГУ.

\section{СПИСОК ЛИТЕРАТУРЫ}

1. Lead Chalcogenides: Physics and Applications. / Ed. by D. Khohlov. New York, London, CRC Press, Taylor \& Francis Group, 2003, 720 p.

2. Infrared Detectors and Emitters: Materials and Devices. / Eds. by Peter Capper and C. T. Elliot. Springer Science + Business Media, LLC, 2001, 498 p. DOI: 10.1007/978-1-4615-1607-1

3. Rogalski A. Infrared Detectors, Second Edition. CRC Press. Taylor \& Francis Group, 2011, 898 p. DOI: 10.1201/ b10319

4. Popescu A., Woods L. M. // Appl. Phys. Lett., 2010, vol. 97, pp. 052102-01-052102-03. DOI: 10.1063/1. 3464288

5. Shaibal Mukherjee, Donghui Li, Anurag Gautam, Jyoti P. Kar, Zhisheng Shi. Lead Salt Thin Film Semiconductors for Microelectronic Applications. Transworld Research Network, Kerala, India, 2010, 88 p.

6. Haotian Fan, Taichao Su, Hongtao Li, Shangsheng Li, Meihua Hu, Bingguo Liu, Baoli Du, Hongan Ma, Xiaopeng Jia. // J. of Alloys and Compounds, 2016, vol. 658, pp. 885-890. DOI: 10.1016/j.jallcom.2015.10.021 
7. Zogg H., Arnold M., Felder F., Rahim M., Fill M., Boye D. // J. Electronic Materials, 2008, vol. 37, № 9, pp. 1497-1503. DOI: 10.1117/12.797849

8. Samoylov A. M., Belenko S. V., Sharov M. K., Dolgopolova E. A., Zlomanov V. P. // J. Cryst. Growth, 2012, vol. 351, pp. 149-154. DOI: 10.1016/j.jcrysgro. 2012.01.042

9. Рябова Л. И., Хохлов Д. Р. // Успехи физических наук, 2014, т. 184, № 10, с. 1033-1044. DOI: $10.3367 /$ UFNe.0184.201410b.1033

10. Harman T. C., Taylor P. J., Walsh M. P., La Forge B. E. // Science, 2002, № 297, pp. 2229-2232. DOI: 10.1126/ science. 1072886

11. Gelbstein Y., Dashevsky Z., Dariel M. P. // Physica B: Condens. Matter., 2005, vol. 363, pp. 196-205. DOI: 10.1016/j.physb.2005.03.022

12. Li J. Q., Lu Z. W., Li S. M., Liu F. S., Ao W. Q., Li Y. // Scripta Materialia, 2016, vol. 112, pp. 144-147. DOI: 10.1016/j.scriptamat.2015.09.036

13. Zogg H. Arnold M. // Opto-Electronics Rev., 2006, vol. 14, № 1, pp. 33-36. DOI: 10.2478/s11772-006-0005-1

14. Самойлов А. М., Беленко С. В., Сирадзе Б. А., Тореев А. С., Донцов А. И., Филонова И. В. // Конденсированные среды и межфазныле границы, 2013, т. 15, № 3, с. 322-331.

15. Акимов А. Н., Климов А. Э., Самойлов А. М., Шумский В. Н., Эпов В. С. // Конденсированные среды и межфазные гранииы, 2013, т. 15, № 4, с. 375-381.
16. Hassan S., Gremenok V. F., Ivanov V. A. // Problems of Physics, Mathematics and Techniques, 2014, vol. 18, № 2 . pp. 26-30. DOI: 10.1002/crat.201000440

17. Ugai Ya. A., Samoylov A. M., Sharov M. K., Tadeev A. V. // Thin Solid Films. 1998, vol. 336, pp. 196200. DOI: 10.1016/s0040-6090(98)01278-4

18. Михайлов В. И., Волков В. Т., Еременко В. Г. // Поверхность. Рентгеновские, синхротронные и нейтронные исследования, 2011, № 6, с. 97-102. DOI: org/10.1134/S1027451011060139

19. Угай Я. А., Самойлов А. М., Агапов Б. А., Долгополова Э. А., Шаров М. К. // Неорган. материальl,1998, Тт. 34, № 9, с. 1048-1054. DOI https://doi. org/10.1023/A:1015410703238

20. Самойлов А. М. Направленный синтез легированных галлием и индием пленок теллурида свинца с контролируемым содержанием примесных атомов $u$ отклонением от стехиометрии. Автореф. дис. ... док. хим. наук. Воронежский государственный университет, Воронеж, 2006, 44 c.

21. JCPDS - International Centre for Diffraction Data [Электронные ресурсы]. (C) 1987-2008. JCPDS-ICDD. Newtown Square, PA 19073. USA. DOI: org/10.1017/ s0885715600011325

22. Ryabtsev S. V., Ievlev V. M., Samoylov A. M., Kuschev S. B., Soldatenko S. A. // Thin Solid Films, 2017, vol. 636, pp. 751-759. DOI:10.1016/j.tsf.2017.04.009

\title{
SURFACE MORPHOLOGY OF PbTe/Si (100) FILMS SYNTHESIZED BY MODIFIED "HOT WALL" EPITAXY TECHNIQUE
}

\author{
(C) 2018 A. M. Samoylov ${ }^{1}$, O. G. Kuzminykh', Yu. V. Synorov², S. A. Ivkov' ${ }^{1}$ B. L. Agapov ${ }^{1}$, \\ E. K. Belonogov ${ }^{3,1}$ \\ ${ }^{1}$ Voronezh State University, 1 Universitetskaya pl., 394018 Voronezh, Russia \\ e-mail:samoylov@chem.vsu.ru \\ ${ }^{2}$ Voronezh State University of Engineering Technologies, pr. Revolyutsii 19, 394036 Voronezh, Russia \\ ${ }^{3}$ Voronezh State Technical University, 14 Moskovskii pr., 394018 Voronezh, Russia
}

Received 12.01.2018

\begin{abstract}
The narrow band gap lead chalcogenide semiconductors and related solid solutions are capable to detect effectively an infrared (IR) radiation in the wavelength range 3-25 $\mu \mathrm{m}$. Due to the unique combination of the optimal dielectric permittivity, Seebeck coefficient, and high thermal conductivity, lead telluride is promising material for manufacturing of high-performance intermediate-temperature thermoelectric devices. By scanning electron microscopy (SEM), electron probe microanalysis (EPMA), X-ray analysis (XRD), and atomic force microscopy (AFM) the quantitative elemental composition, phase nature, surface morphology, and average surface roughness of homogeneous lead telluride films synthesized by modified «hot wall» technique on $\mathrm{Si}$ (100) substrates have been studied. The XRD patterns have shown that at condition of high condensation process rate, which was realized at substrate temperatures below $\mathrm{T}=573 \mathrm{~K}$ and corresponded to the maximum deviation from the thermodynamic equilibrium, polycrystalline PdTe films on Si (100) substrates were synthesized only. The increase in temperature $\mathrm{Si}(100)$ substrate to $\mathrm{T}=593-613 \mathrm{~K}$ has led to fabrication of mosaic single-crystal PdTe films with (100) texture. By SEM and AFM methods it has been found that under constant values of lead and tellurium vapour pressure synthesis an increase in
\end{abstract}


the Si substrate temperature led to increase in crystallite average lateral dimension of PbTe film on Si (100) substrates. By AFM method it has established the tendency of the average surface roughness value increasing with average thickness for both polycrystalline and mosaic single crystal $\mathrm{PbTe} / \mathrm{Si}$ (100) films. At the same average thickness values polycrystalline $\mathrm{PbTe} / \mathrm{Si}$ (100) films are characterized by higher surface roughness compared to mosaic single-crystal PbTe (100) samples. It is suggested that of average surface roughness-to-film thickness ratio it can be used as a criterion of evaluation of surface contribution to functional properties of PbTe films. It has been shown that with optimum values of average roughness-to-thickness ratio mosaic single-crystal PdTe (100) films with thickness over $1.2 \mu \mathrm{m}$ are characterized.

Keywords: lead telluride, thin films, microstructure, surface morphology, crystallite average lateral dimension, surface roughness.

DOI: https://doi.org/10.17308/kcmf.2018.20/483

\section{ACKNOWLEDGEMENTS}

The research was carried out using the equipment of the Centre for Collective Use of Scientific Equipment of Voronezh State University.

\section{REFERENCES}

1. Lead Chalcogenides: Physics and Applications / Ed. by D. Khohlov, S. Furusaki, J. Garside. New York, London, CRC Press. Taylor \& Francis Group, 2003, 720 p.

2. Infrared Detectors and Emitters: Materials and Devices / Eds. by Peter Capper and C. T. Elliot. Springer Science + Business Media, LLC, 2001, 498 p. DOI: 10.1007/978$1-4615-1607-1$

3. Rogalski A. Infrared Detectors, Second Edition. CRC Press. Taylor \& Francis Group, 2011, 898 p. DOI: 10.1201/ b10319

4. Popescu A., Woods L. M. Appl. Phys. Lett., 2010, vol. 97, pp. 052102-01-052102-03. DOI: 10.1063/ 1.3464288

5. Shaibal Mukherjee, Donghui Li, Anurag Gautam, Jyoti P. Kar, Zhisheng Shi. Lead Salt Thin Film Semiconductors for Microelectronic Applications. Transworld Research Network, Kerala, India, 2010, 88 p.

6. Haotian Fan, Taichao Su, Hongtao Li, Shangsheng Li, Meihua Hu, Bingguo Liu, Baoli Du, Hongan Ma, Xiaopeng Jia. J. of Alloys and Compounds, 2016, vol. 658, pp. 885-890. DOI: 10.1016/j.jallcom.2015.10.021

7. Zogg H., Arnold M., Felder F., Rahim M., Fill M., Boye D. J. Electronic Materials, 2008, vol. 37, no. 9, pp. 1497-1503. DOI: 10.1117/12.797849

8. Samoylov A. M., Belenko S. V., Sharov M. K., Dolgopolova E. A., Zlomanov V. P. J. Cryst. Growth, 2012, vol. 351, pp. 149-154. DOI: 10.1016/j.jcrysgro.2012.01.042

9. Ryabova L. I., Khokhlov D. R. Physics-Uspekhi, 2014, vol. 57, no. 10, pp. 959-969. DOI: $10.3367 /$ UFNe.0184.201410b.1033

10. Harman T. C., Taylor P. J., Walsh M. P., La Forge B. E. Science, 2002, vol. 297, pp. 2229-2232. DOI: 10.1126/science. 1072886

11. Gelbstein Y., Dashevsky Z., Dariel M. P. Physica B: Condens. Matter., 2005, vol. 363, pp. 196-205. DOI: 10.1016/j.physb.2005.03.022
12. Li J. Q., Lu Z. W., Li S. M., Liu F. S., Ao W. Q., Li Y. Scripta Materialia, 2016, vol. 112, pp. 144-147. DOI: 10.1016/j.scriptamat.2015.09.036

13. Zogg H. Arnold M. Opto-Electronics Rev., 2006, vol. 14, no. 1, pp. 33-36. DOI: 10.2478/s11772-006-00051

14. Samoylov A. M., Belenko S. V., Siradze B. A., Toreev A. S., Dontsov A. I., Filonova I. V. Condensed Matter and Interphases, 2013, vol. 15, no. 3, pp. 322-331. Available at: http://www.kcmf.vsu.ru/resources/t_15_3_ 2013_016.pdf (in Russ.)

15. Akimov A. N., Klimov A. E., Samoylov A. M., Shumsky V. N., Epov V. S. Condensed Matter and Interphases, 2013, vol. 15, no. 4, pp. 375-381. Available at: http://www.kcmf.vsu.ru/resources/t_15_4_2013_001.pdf (in Russ.)

16. Hassan S., Gremenok V. F., Ivanov V. A. Problems of Physics, Mathematics and Techniques, 2014, vol. 18, no. 2, pp. 26-30. DOI: 10.1002/crat.201000440

17. Ugai Ya. A., Samoylov A. M., Sharov M. K., Tadeev A. V. Thin Solid Films, 1998, vol. 336, pp. 196-200. DOI: 10.1016/s0040-6090(98)01278-4

18. Mikhailov V. I., Volkov V. T., Eremenko V. G., Kanevsky V. M., Polyak L. E., Rakova E. V., Muslimov A. E., Kvartalov V. B. Surface. X-ray, Synchrotron and Neutron Studies, 2011, no. 6, pp. 97-102. DOI: org/10.1134/ S1027451011060139

19. Ugai Ya., Samoilov A. M., Agapov B. A., Dolgopolova E. A., Sharov M. K. Inorganic Materials, 1998, vol. 34, no. 9, pp. 873-877. DOI https://doi.org/10.1023/ A: 1015410703238

20. Samoylov A. M. Directed Synthesis of Lead Telluride Thin Films Doped with Gallium and Indium with a Controlled Content of Impurity Atoms and Deviation from Stoichiometry. Doct. (Chem.) Diss. Thesis. Voronezh State University, Voronezh, 2006, 44 p.

21. JCPDS - International Centre for Diffraction Data. 1986-2008. JCPDS-ICDD. Newtown Square, PA 19073. USA. DOI: org/10.1017/s0885715600011325

22. Ryabtsev S. V., Ievlev V. M., Samoylov A. M., Kuschev S. B., Soldatenko S. A. Thin Solid Films, 2017, vol. 636, pp. 751-759. DOI:10.1016/j.tsf.2017.04.009 
Самойлов Александр Михайлович - д. х. н., профессор кафедры материаловедения и индустрии наносистем, Воронежский государственный университет; тел.: +7(473) 2596515, e-mail: samoylov@chem.vsu.ru

Кузьминых Олег Геннадьевич - магистрант кафедры материаловедения и индустрии наносистем, Воронежский государственный университет, kuzminykh.oleg@mail.ru

Сыноров Юрий Владимирович - доцент, кафедра физики, Воронежский государственный университет инженерных технологий; e-mail: synor@mail.ru

Ивков Сергей Александрович - аспирант, ведущий электроник кафедры физики твердого тела и наноструктур, Воронежский государственный университет; тел.: +7 (473) 2208363, e-mail: ftt@phys.vsu.ru

Агапов Борис Львович - к. т. н., ЦКПНО, Воронежский государственный университет

Белоногов Евгений Константинович - д. ф.-м. Н., профессор, Воронежский государственный технический университет, Воронежский государственный университет; тел.: +7(473) 2467633, e-mail: ekbelonogov@mail.ru
Alexandr M. Samoylov - Dr. Sci. (Chem.), Professor of the Department of Materials Science and Industry of Nanosystems, Voronezh State University; tel.: +7(473) 2596515, e-mail: samoylov@chem.vsu.ru

Oleg G. Kuzminykh - master degree student of the Department of Materials Science and Industry of Nanosystems, Voronezh State University; kuzminykh.oleg@mail.ru

Yurii V. Synorov - Associate Professor, Voronezh State University of Engineering Technologies; e-mail: synor@ mail.ru

Sergey A. Ivkov - postgraduate student, Leading Electronics of Solid State Physics and Nanostructures Department, Voronezh State University; tel.: +7 (473) 2208363, e-mail: ftt@phys.vsu.ru

Boris L. Agapov - Cand. Sci. (Eng.), Centre for Collective Use of Scientific Equipment, Voronezh State University

Evgenii K. Belonogov - Dr. Sci. (Phys.-Math.), Full Professor, Voronezh State Technical University, Voronezh State University; tel.: +7(473) 2467633, e-mail: ekbelonogov@mail.ru 